\title{
Die simultane Bestimmung der verschiedenen Körperflüssigkeitsräume ${ }^{1}$
}

\author{
Von A. Boner und W. Siegenthaler \\ Aus der Medizinischen Universitäts-Poliklinik Zürich (Direktor: Prof. Dr. R. Hegglin)
}

(Eingegangen am 16. Januar 1969)

Es wird über die simultane Volumenbestimmung der verschiedenen Körperflüssigkeitstäume mit Evans-blue und Isotopen berichtet. Die Frage der Homogenität des durch das Verdünnungsprinzip zu untersuchenden Milieus wird kritisch beleuchtet. Anhand von Fällen, die vor und nach einer die zirkulatorischen Verhältnisse stark beeinflussenden Therapie untersucht wurden, wird festgestellt, daß sich beim einzelnen Individuum gewisse Korrelationen zwischen den verschiedenen Verteilungstäumen erkennen lassen. Allgemein gültige Regeln lassen sich jedoch nicht aufstellen.

\section{The simultaneous. determination of different body fluid spaces}

Body fluid volumes have been studied simultaneously with Evans-blue and isotopes. In any dilution method repartition must be complete before sampling. This point has been closely investigated. Cases with circulatory disorders have been examined before and after effective therapyr. No general rule could be drawn. Nevertheless there is a certain correlation between the different spaces in the individual cases.

Im Rahmen unserer Studien über die Regulation des Wasserhaushaltes (13) und im Hinblick auf Untersuchungen über das Verhalten der Körperflüssigkeitsräume bei verschiedenen Krankheiten, suchten wir nach Möglichkeiten, die uns eine gleichzeitige Bestimmung der verschiedenen Flüssigkeitsräume des Organismus gestatten würden.

Dabei verwendeten wir Evans blue (T 1824) zur Erfassung des Plasmavolumens (2), Tritiumwasser $\left({ }^{3} \mathrm{H}_{2} \mathrm{O}\right)$ für das Gesamtkörperwasser $(1-15)$ und Radiosulfat $\left(\mathrm{Na}_{2}{ }^{35} \mathrm{SO}_{4}\right)$ für den Extrazellulärraum (16-20). Durch Substraktion der mit radioaktivem Natriumsulfat für den Extrazellulärraum ermittelten Werte von den für das Gesamtkörperwasser bestimmten Größen läßt sich die im intrazellulären Raum vorhandene Flüssigkeitsmenge berechnen.

Das Prinzip der Körperflüssigkeitsbestimmung beruht für alle Räume auf einer Verdünnungsmessung. Der injizierte Farbstoff- oder Isotopenindikator muß sich zuerst homogen in dem zu untersuchenden Volumen verteilen. $\mathrm{Da}$ der dafür adäquate Zeitpunkt nicht zum voraus bekannt ist, haben wir die Blutentnahmezeiten so gestaffelt, daß dieser in jedem Fall ermittelt werden kann.

Zur Bestimmung des Plasmavolumens haben wir Evans blue (T 1824) gewählt. Es handelt sich dabei um einen Farbstoff, der sich mit dem Plasmaeiweiß verbindet und leicht photometrisch bestimmt werden kann. Diese Substanz wird seit Jahren mit gutem Erfolg auch zur Kreislaufzeitbestimmung benützt.

Die Bestimmung des Gesamtkörperwassers wurde mit Tritiumwasser, die des Extrazellulärraumes mit ${ }^{35} \mathrm{~S}$ markiertem Natriumsulfat durchgeführt. Diese beiden. unter den $\beta$-Strahlern figurierenden Isotopen wurden bei unseren Untersuchungen wegen der durch den

\footnotetext{
1) Diese Arbeit wurde durch die Hilfe des Schweizcrischen Nationalfonds zur Förderung der wissenschaftlichen Forschung und die Stiftung für wissenschaftliche Forschung an der Universität Zürich ermöglicht.
}

Energieunterschied gegebenen Möglichkeit einer Simultanbestimmung gewählt. Dazu verwendeten wir ein Tri-carb liquid scintillation Spectrometer. Außerdem sind die beiden Substanzen in der Handhabung äußerst bequem, da sie dank ihrer Halbwertszeiten von 12,26 Jahren bzw. 87 Tagen auch bei nicht sehr häufigen Bestimmungen ein weitgehend verlustfreies Arbeiten exlauben.

\section{Methodik}

Das Evans blue Verteilungsvolumen wurde mit Plasmaproben bestimmt, die in Zeitabständen zwischen 2 und $60 \mathrm{Min}$. nach i. v. Injektion des Farbstoffes (T 1824) entnommen wurden. Mit Leerplasma des Patienten, dem verschiedene genau bekannte Mengen des Farbstoffes zugefügt wurden (Standardverdünnungsreihe) wurde anschließend ein photometrischer Vergleich angestellt (Beckman DBl Spectrophotometer, bei $625 \mathrm{~nm}$ ). Wir injizierten etwa $0,01 \mathrm{ml}$ der 2proz. Evans blue Lösung pro kg Körpergewicht. Die Ermittlung der genauen Menge erfolgte gravimetrisch. Die Ausdehnung der Blutentnahmen über die Zeitspanne von $60 \mathrm{Min}$. erwies sich infolge der bei Herzinsuffizienz festgestellten Verzögerung der Durchmischung bei diesem Krankheitsbild als notwendig (21).

Die zur Gesamtkörperwasserbestimmung und zur Erfassung des extrazellulären Wassers verwendeten Isotopen wurden vom Schweizerischen Institut für Reaktorforschung in Würenlingen hergestellt, so Tritiumwasser $\left({ }^{3} \mathrm{H}_{2} \mathrm{O}\right)$ in physiol. $\mathrm{NaCl}$-Lösung, steril und pyrogenfrei mit einer spezifischen Aktivität von $500 \mu \mathrm{c} / \mathrm{m} /$. Pro kg Körpergewicht wurden $20 \mu \mathrm{c}$ i. v. verabreicht. Vom $\mathrm{Na}_{2}{ }^{35} \mathrm{SO}_{4}$ in physiol. $\mathrm{NaCl}-\mathrm{Lösung}$, steril und pyrogenfrei mit einer spezifischen Aktivität von $50 \mu \mathrm{c}$ pro $\mathrm{ml}$ wurden pro kg Körpergewicht $2 \mu \mathrm{c} \mathrm{i}$. v. verabreicht. Die spezifischen Aktivitäten wurden so gewählt, daß zur Injektion keine Verdünnungen mehr hergestellt werden mußten. Die verwendeten Substanzen verursachten nie irgendwelche Reaktionen.

Von diesen Isotopen wurden zudem 1:2000 Standardlösungen hergestellt, die wie das Blut verarbeitet wurden. Sie dienten auch zur Kontrolle der Einstellung des Apparates.

Die Anwendung dieser Substanzen am Patienten erfolgte folgendermaßen: $1 / 2$ Stunde vor Beginn des Versuches bekam der nüchtcrne Patient zur Aufrechterhaltung der Diurese $1 / 2$ Liter warmen Lindenblütentee zu trinken. An einem Arm wurde eine Infusion mit physiol. NaCl-Lösung gesteckt, die lediglich zur Sicherung des Injektionsweges diente und die wir deshalb mit minimaler Geschwindigkeit laufen ließcn. Am anderen Arm wurde cine 
venösc Vcrweilkanüle gesteckt, die mit Hilfe von Heparin durchgängig gehalten wurde. Bei einem Teil der Patienten wurde zudem ein Blasenkatheter eingelegt. Nach Entnahme der Blut- und Urinleerwerte wurden in den Infusionsschlauch unterhalb einer Abklemmung nadelnahe zuerst $0,01 \mathrm{~m} / 2$ proz. Evans blue pro kg Körpergewicht und sofort anschließend gleichzeitig aus 2 verschiedenen Spritzen die beiden Isotopen ${ }^{3} \mathrm{H}_{2} \mathrm{O} 20 \mu \mathrm{c}$ pro $\mathrm{kg}$ Körpergewicht und $\mathrm{Na}_{2}{ }^{35} \mathrm{SO}_{4} 2 \mu \mathrm{c}$ pro kg Körpergewicht injiziert. Die Spritzen wurden vor und nach Applikation gewogen. Für die Isotopen wurden Einmal-Spritzen verwendet. Nach erfolgter Injektion ließen wir die Infusion sofort $1 \mathrm{Min}$. lang rasch laufen, dann wurde sie entfernt. In den ersten $3 \mathrm{Stdn}$. wurde stdl. ein Glas Tee gegeben, nachher normales Essen mit genügender Flüssigkeitszufuhr verabreicht. $\mathrm{Da}$ das Gleichbleiben von Luftfeuchtigkeit und Temperatur nicht gewährleistet werden konnte, haben wir auf eine konstante Flüssigkeitszufuhr verzichtet. Die Genauigkeit der Tritiumwasserbestimmung hätte dadurch nicht wesentlich gesteigert werden können.

Die Bestimmung der Tritiumwvasser- und Radiosulfat-Aktivität nach i. $\nabla$. Verabreichung von ${ }^{3} \mathrm{H}_{2} \mathrm{O}$ und $\mathrm{Na}_{2}{ }^{35} \mathrm{SO}_{4}$ erfolgte im Plasmawasser in Zeitabständen zwischen $2 \mathrm{Min}$. und $72 \mathrm{Stdn}$. Um Bilanzstudien durchzuführen, wurde parallel dazu auch die Ausscheidung im Urin bestimmt, während die Ausscheidung im Stuhl vernachlässigt werden konnte (8).

Zur Erfassung der ${ }^{3} \mathrm{H}_{2} \mathrm{O}$ und $\mathrm{Na}_{2}{ }^{35} \mathrm{SO}_{4}$ Radioaktivität im Blut sind pro Bestimmung $2 \mathrm{~m} /$ Plasma notwendig. Das Eiweiß wird mit $1 \mathrm{ml} 20$ prò. Trichloressigsäure gefällt. Daraufhin wird zweimal abzentrifugiert und vom Uberstand $0,5 \mathrm{~m} /$ in $10 \mathrm{~m} l$ Scintillationsflüssigkeit folgender Zusammensetzung gebracht (22):

7 g PPO (2,5-Diphenyloxazol) scintillation grade Packard,

$50 \mathrm{mg}$ Dimethyl-POPOP (1,4-Bis-2-(4methyl-5phenoxyloxazolylbenzol)) scintillation grade Packard,

$50 \mathrm{~g}$ Naphtalin (organic analytical standard) British Drug Houses, ad 1000 Dioxan purissimum Fluka.

Ein Zusatz von 7,5\% Methanol erwies sich als notwendig, um ein Einfrieren während der Messung in der gekühlten Zählkammer zu verhindern. Der dadurch exlittene Ausbeuteverlust hielt sich in annehmbaren Grenzen. Die Zählung von ${ }^{3} \mathrm{H}$ und ${ }^{35} \mathrm{~S}$ erfolgte simultan im Packard liquid scintillation Spectrometer (series $314 \mathrm{E}$ ) bis zu 10000 Impulsen ${ }^{35} \mathrm{~S}$ bzw. bis zu 100000 Impulsen ${ }^{3} \mathrm{H}$, oder über $30 \mathrm{Min}$.

Zur Bestimmung der Tritium- und Sulfat-Radioaktivität im Urin wurde $1 \mathrm{~m} l$ Urin mit $4 \mathrm{~m} /$ Wasser verdünnt. Davon wurden $2 \mathrm{~m} l$ mit $2 \mathrm{~m} / 20$ proz. Trichloressigsäure versetzt. Obwohl dieser $\mathrm{Zu}-$ satz nur bei Proteinurie notwendig ist, wird er bei jeder Bestimmung gegeben, um konstante Zählverhältnisse zu erhalten. Wenn nötig wird zentrifugiert und $0,5 \mathrm{~m} l$ des Uberstandes in $10 \mathrm{~m} l$ Scintillationsflüssigkeit gegeben. Die Verdünnung dient dor Verminderung einer eventuellen Störung durch die im Urin enthaltenen Farbstoffe.

Das Plasmavolumen läßt sich graphisch ermitteln. Auf der Ordinate werden die Extinktionswerte, auf der Abszisse die entsprechenden Verdünnungskoeffizienten der Standardverdünnungen aufgetragen und die Plasmawerte mit der Eichkurve verglichen. $\mathrm{Da}$ die Linearität keine absolute ist, müssen genügend $Z$ wischenwerte vorhanden sein, so daß beim Ablesen der Plasmaproben nur auf einem kurzen Bereich extrapoliert werden muß, wodurch der entstehende Febler möglichst klein gehalten werden kann.

Das Gesamtkörperwasser berechnet sich folgendermaßen:

$\mathrm{V}($ Volumen $)=\frac{\text { Total der injizierten }{ }^{3} \mathrm{H}-\text { Impulse }}{{ }^{3} \mathrm{H}-\text { Impulse pro } \mathrm{m} / \text { Plasma } \cdot 1,075}=\begin{aligned} & \mathrm{xm} / \text { oder } \\ & \text { X\% vom } \\ & \text { Körper- } \\ & \text { gewicht }\end{aligned}$

(1,075 Korrektur für die Plasmaproteine).

Es wurden der 90 Minuten-, der 3 Stunden-, sowie der Wert bei Erreichung des Gleichgewichtes berücksichtigt. Die Ausscheidung im Urin war in diesen Zeitabständen gering und konnte vernachlässigt werden.
Die Größe des Extrazellulärraumies wird folgendcrmaßen bestimmt: ${ }^{35} \mathrm{SO}_{4}$ wird auf die Zeit Null zurückberechnet (16). Die 30-, (60-) und 90 Minuten Plasmawerte werden auf $t_{0} 1 / 2$ logarithmisch extrapoliert $=\alpha$. Total injizierte Menge $t_{0}$ - ausgeschiedene Menge im Urin nach 30-, (60-) und 90 Minuten auf $t_{0} 1 / 2$ logarithmisch extrapoliert $=\beta$. Diese Korrektur erfolgt, da die ${ }^{35} \mathrm{SO}_{4}$-Ausscheidung in diesem Zeitraum beträchtlich ist.

$\mathrm{V}($ Volumen $)=\frac{\beta}{\alpha \times 1,194}=\mathrm{y} \mathrm{m} l$ oder $Y \%$ vom Körpergewicht (1,194 Korrektur für die Plasmaproteine und den Donnan-GibbsEffekt).

Falls nur das Verteilungsvolumen und kein Verlauf zu ermitteln ist, kann man auf die Urinausscheidung verzichten und die total injizierte Menge in die Formel einsetzen. Innerhalb dieses kurzen Zeitabstandes verhält sịch die Ausscheidung wie eine einfach exponentielle Funktion. In diesem Fall ergibt sich folgende Berechnungsformel:

$$
\mathrm{V}=\frac{\text { Total der injizierten Impulse }}{\alpha \times 1,194} .
$$

Zusätzlich haben wir noch die Plasmaaktivität der Isotopen in Zusammenhang mit dem Plasmavolumen gebracht unter Berücksichtigung der Ausscheidung. Dies erlaubte uns genauexe Plasmaaktivitätskurven aufzustellen. Anhand von diesen wurden dann die Durchmischungszeit und in speziellen Fällen die Halbwertszeit bestimmt.

Der im Organismus zu jedem Zeitpunkt verbleibende Restbetrag der injizierten tadioaktiven Menge wird unter Berücksichtigung der Ausscheidung durch den Harn berechnet und in \% der verabreichten Menge ausgedrückt. Die zeitlich entsprechende Plasmaaktivität pro $\mathrm{m} l$ wird durch die im Körper verbliebene Menge (in $\%$ der injizierten Menge ausgedrückt) dividiert und mit 100 multipliziert, so daß die am Austausch beteiligte Menge immer als $100 \%$ betrachtet werden kann (mathematische Nephrektomie). Die Plasmaaktivität im Zeitpunkt Null berechnet sich folgendermaßen:

$$
\text { Impulse pro } \mathrm{m} l \mathrm{t}_{0}=100 \%=\frac{\text { Total injizierte Impulse }}{\text { Plasmavolumen in } \mathrm{m} l} .
$$

Die im Plasma zurückgebliebene Menge wird gegeben durch:

$\%$ effektiv $\mathrm{t}_{\mathbf{x}}=\frac{\left(\text { Impulse pro } \mathrm{m} / \mathrm{Plasmaentnahme} \mathrm{t}_{\mathbf{x}}\right) \times 100 \times 100}{\left(\text { Impulse pro } \mathrm{m} / \mathrm{t}_{\mathbf{0}}\right) \cdot(\text { im Körper retinierte Akti- }}$ vität in $\%$ der injizierten Mengè)

Damit wird die Ausscheidung, die über längere Zeitabstände unregelmäßig exfolgt und mathematisch nicht sicher erfaßbar ist, mitberücksichtigt. Sie spielt bei der Berechnung des Gesamtkörperwassers mit ${ }^{3} \mathrm{H}$ eine unbedeutende Rolle. Die auf diese Weise erhaltenen Kurvenverläufe werden in Abbildung 1 und 2 gezeigt.

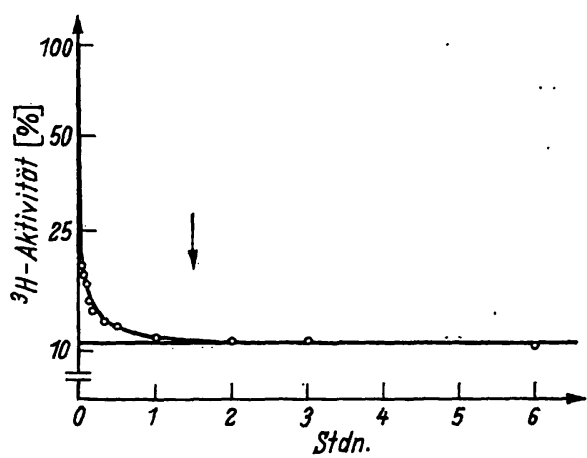

Abb. 1

Graphische Ermittlung der Tritium-Wasser-Durchmischungszeit im Gesamtkörper-Wasser

(Die Aktivität in jedem Zeitpunkt ist: in \% der Aktivität pro $\mathrm{ml}$ Plasma $t_{0}$ angegeben) (Fall 20) 


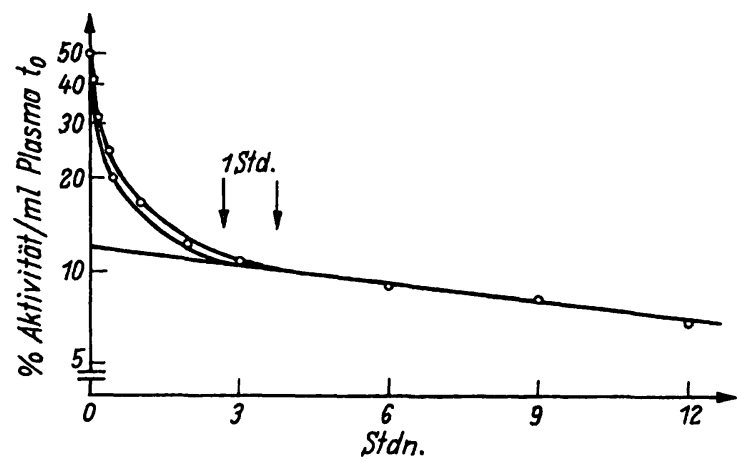

Abb. 2

Graphische Analyse der Aktivität nach i. v. Applikation von $\mathrm{Na}_{2}{ }^{36 \mathrm{SO}}$ Werte für Urinausscheidung korrigiert; Werte in \% der Aktivität pro $\mathrm{m} l$ Plasma $\mathrm{t}_{0}$ (Fall 22)

\section{Resultate}

Wir versuchten zunächst die von uns erhaltenen Werte für die verschiedenen Körperflüssigkeitsräume mit den in der Literatur dafür angegebenen Normalwerten zu vergleichen. Die Plasmavolumenbestimmung ergab im -allgemeinen Werte im erwarteten Bereich. Der Normbereich für das Blutvolumen liegt bei 53-85 resp. $69 \pm 8 \mathrm{ml}$ Blut pro kg Körpergewicht (21). Bei der Gesantkörperwasser- und Extrazellulärraumbestimmung traten dagegen so große Streuungen auf, daß nur etwa $50 \%$ der Werte im erwarteten Bereich lagen, wobei diese Abweichungen sowohl bei den Probanden mit gesundem Körperflüssigkeitshaushalt wie bei den pathologischen Zuständen in ungefähr gleicher Häufigkeit auftraten, also auffallenderweise nicht nur bei gestörtem Wasserhaushalt. Demzufolge scheint diese Art der Auswertung zum Studium der Flüssigkeitsräume recht unbefriedigend.

\section{Diskussion}

Unsere Untersuchungen zur gleichzeitigen Bestimmung der verschiedenen Körperflüsssigkeitsräume haben vor allem für die quantitative Erfassung des Gesamtkörperwassers (GKW) und des extrazellulären Wassers von den erwarteten Resultaten so abweichende Werte ergeben, daß eine exakte Messung dieser Räume auf größte Schwierigkeiten stößt. Daran scheịnen verschiedene Gründe beteiligt zu sein. Tatsache ist, daß bei solchen Untersuchungen von der Methode der Mumifikation bis zur Isotopenverdünnung keine entscheidenden Fortschritte gemacht worden sind. Das Plasmavolumen hingegen ist besser bestimmbar und kann als Bezugsgröße herangezogen werden. Die relativ weiten Normbereiche für das Gesamtkörperwasser $(45-75 \%$ des Körpergewichtes) $(5,8)$ und für den Extrazellulärraum (10-27\% des Körpergẹwichtes $(18,19))$ sowie die Tatsache, daß wir bei ödematösen Patienten den normalen Wassergehalt nicht kennen, schränkt die praktische Anwendung dieser Untersuchungen sowohl für absolute Messungen als auch für Verlaufsstudien stark ein. Man möchte sich PrTts (8) anschließen und den Vorschlag äußern, die Patienten zu wiegen. Unsere Untersuchungen haben gezeigt, daß bei einer technisch befriedigenden Bestimmung der verschiedenen Körperflüssigkeitstäume die Resultate auch bei Verwendung neuer Substanzen nicht besser geworden sind. Wiederholte Bestimmungen bei den gleichen Patienten, jedoch unter anderen zirkulatorischen Bedingungen haben uns erlaubt, interessante Beobachtungen anzustellen, über die wir in dieser Arbeit noch berichten. Zuerst möchten wir noch speziell auf eine Fehlerquelle aufmerksam machen, die durch die Natur der Verdünnungsmethode bedingt ist. Um eine repräsentative Plasmaprobe $z u$ erhalten, müssen wir sicher sein, daß die Durchmischung in dem zu messenden Raum eine homogene ist. Es wurden die Durchmischungszeit für das Evans-blau im Plasmaraum und diejenige für das Tritiumwasser im Gesamtkörperwasser studiert.

\section{Evans-blue Durchmischungszeit im Plasma}

$\mathrm{Zu}$ diesem $\mathrm{Zweck}$ haben wir die Plasmakonzentration des Farbstoffes bis eine Stunde nach der Injektion weiterverfolgt. Eine allzu engmaschige Kontrolle innerhalb - dieser Zeit konnte aus praktischen Gründen (Volumen der Blutentnahmen) nicht durchgeführt werden. Die ersten Werte - alle $2 \mathrm{Min}$. bis 10 Minuten - zeigten sehr große Schwankungen, was der noch mangelhaften Durchmischung zuzuschreiben ist (Abb. 3). Die repräsentative Durchmischungszeit befindet sich im Beginn einer quasilinear abfallenden Kurve. Bei den Werten, die mit weniger als 10 Minuten angegeben sind (Tab. 1) wurden vor diesem Zeitpunkt keine Messungen

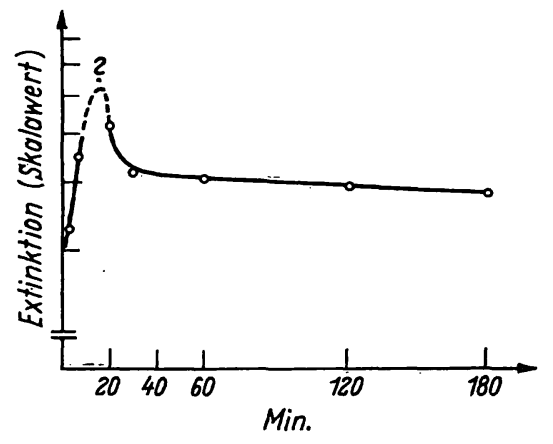

Abb. 3

Evans-blue Durchmischungszeit bei einem Patienten mit dekompensiertem kombinierten rheumatischen Vitium (Fall 21)

Tab. 1

Evans-blue Durchmischungszeit im Plasma bei Herzpatienten und Herzgesunden

\begin{tabular}{|c|c|c|c|}
\hline & $\begin{array}{l}\text { Fall } \\
\text { Nr. }\end{array}$ & Diagnose & $\begin{array}{c}\text { Evans-blue } \\
\text { Durchmischungszeit } \\
\text { (Minuten) }\end{array}$ \\
\hline 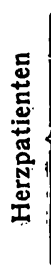 & $\begin{array}{r}2 \\
4 \\
5 \\
8 \\
15 \\
19 \\
21 \\
22 \\
23 \\
24 \\
25\end{array}$ & $\begin{array}{l}\text { Myokardinsuffizienz } \\
\text { Myokardinsuffizienz } \\
\text { Myokardinsuffizienz } \\
\text { Aorteninsuffizienz } \\
\text { rheumat. Mitralinsuffizienz } \\
\text { PCP + dekomp. Hypertonie } \\
\text { dekomp. komb. rheumat. Vitium } \\
\text { dekomp. Aortenstenose } \\
\text { komb. rheumat. Vitium } \\
\text { Aortenstenose } \\
\text { Mitralinsuffizienz }\end{array}$ & $\begin{array}{l}>30 \\
<10 \\
20-30 \\
<10 \\
30-60 \\
<10 \\
30-60 \\
10-20 \\
<101 \\
20-30 \\
6-20\end{array}$ \\
\hline 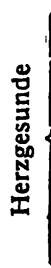 & $\begin{array}{r}3 \\
6 \\
7 \\
10 \\
11 \\
12 \\
14 \\
16 \\
17 \\
18 \\
20\end{array}$ & $\begin{array}{l}\text { Leukämie } \\
\text { Lungenfibrose } \\
\text { Leberpraekoma } \\
\text { Bronchuskarzinom } \\
\text { Asthma } \\
\text { PCP + Oesophaguskarzinom } \\
\text { vegetative Dystonie } \\
\text { Leberechinokokkus } \\
\text { Hypertonie } \\
\text { Neurose } \\
\text { Kollagenose }\end{array}$ & $\begin{array}{l}<10 \\
10-20 \\
10-20 \\
<10 \\
<15 \\
<10 \\
<10 \\
<6 \\
<10 \\
<10 \\
<10\end{array}$ \\
\hline
\end{tabular}


durchgeführt, jedoch 2 minütlich nachher und dabei keine Schwankungen mehr festgestellt.

\section{Resultate}

In Tabelle 1 sind alle untersuchten Fälle aufgeführt. Es wurde eine Aufteilung in Kreislaufgesunde und Kreislaufkranke vorgenommen.

Von 11 Patienten mit nicht manifest gestörtem Kreislauf zeigten 8 eine Evans-blue Durchmischungszeit von weniger als 10 Minuten, einer lag unter 15, zwei bewegten sich $z$ wischen 10 und 20 Minuten.

Von 11 Kreislaufpatienten zeigten 4 eine Durchmischungszeit unter 10 Minuten, 2 eine zwischen 10 respektive 6 und 20 Minuten, 2 eine von 20 bis 30 Minuten, 3 eine von 30 bis 60 Minuten, respektive über 30 Minuten.

\section{Diskussion}

Bei den meisten Kreislaufgesunden ist die Durchmischung innerhalb 10 Minuten erreicht. Die Patienten mit erhöhten Werten - bis 20 Minuten - litten an Lungenfibrose, Leberpräkoma, Asthma, wobei klinisch keine Insuffizienzzeichen von Seiten des Kreislaufs festgestellt werden konnten.

Bei kreislaufgeschädigten Patienten fanden wir eine große Streubreite mit zum Teil wider Erwarten niedrigen Zeiten. Ein niedriger Wert wurde auch bei einer Herzinsuffizienz (Myodegeneratio et insufficientia cordis) mit Ödemen, ein anderer bei einer präterminalen Aorteninsuffizienz ohne periphere Ödeme beobachtet. Inwieweit hier das Schlagvolumen die Durchmischungszeit beeinflußt, muß offen bleiben.

\section{Tritiumdurchmischungszeit im Gesamtkörper- wasser}

In ähnlicher Weise haben wir die Tritiumwasserdurchmischungszeit beobachtet. Um vergleichbare Resultate zu erhalten, haben wir die Durchmischungszeit in Stunden pro Liter Gesamtkörperwasser angegeben. Dies erfolgte um Unterschiede der individuellen Konstitution zu berücksichtigen.

\section{Resultate}

In Tabelle 2 haben wir die Ergebnisse bei Herzpatienten dargestellt, in Tabelle 3 die bei Kreislaufgesunden, die zusätzlich noch in Altersklassen aufgeteilt wurden. Es
Tab. 2

Durchmischungszeit pro Liter Gesamtkörperwasser bei Herzpatienten TDZ Std. $/ l=$ Tritium-Wasser-Durchmischungszeit in Stunden pro Liter Gesamtkörperwasser

\begin{tabular}{clc}
\hline Fall Nr. & \multicolumn{1}{c}{ Diagnose } & TDZ Std./l \\
\hline 24 & komp. Aortenstenose & 0,234 \\
8 & komp. Aorteninsuffizienz & 0,087 \\
25 & komp. Mitralinsuffizienz & 0,028 \\
15 & komp. rheumat. Mitralinsuffizienz & 0,057 \\
23 & komp. komb. rheumat. Vitium & 0,081 \\
22 & dekomp. Aortenstenose & 0,135 \\
21 & dekomp. komb. rheumat. Vitium & 0,144 \\
4 & Myokardinsuffizienz & 0,068 \\
5 & Myokardinsuffizienz & 0,073 \\
\hline
\end{tabular}

ist daraus ersichtlich, daß die festgestellten Werte eine große Streubreite aufweisen, und zwar bei den Kreislaufgesunden von $0,047 \mathrm{Std} / l$ bis $0,150 \mathrm{Std} / l$, bei Herzpatienten von 0,028 bis $0,234 \mathrm{Std} / l$.

\section{Diskussion}

Erstaunlich ist, daß bei den Herzpatienten im Vergleich zum Gesunden die Streuung nicht nur wie erwartet durch die größere Flüssigkeitsmenge und verlängerte Kreislaufzeit nach oben, sondern auch nach unten ausgesprochener ist. Man ist daher zur Annahme berechtigt, daß in letzterem Fall Zustände mit besonders rascher Diffusionsgeschwindigkeit für Wasser bestehen. Andererseits können, da Wasser rasch diffundiert, übermäßig erhöhte Werte wiederum nicht durch Kreislauffaktoren allein, sondern auch durch Permeabilitätsfaktoren erklärt werden. Dies gilt nur für Patienten ohne Ergüsse oder Ascites. Es ist auffallend, $\mathrm{da} B$ diese Streuung nach oben und unten bei Patienten mit verschiedensten Herzaffektionen beobachtet wurde (Tab. 2). Bei den Kreislaufgesunden fanden wir den niedrigsten Wert (Std/l) bei einem 24jährigen Patienten mit einem Leberechinococcus, den höchsten bei einem 59jährigen Asthmatiker. Eine Korrelation mit dem Alter konnte nicht gefunden werden (Tab. 3). Jeder Patient scheint somit seinen individuellen Wert zu haben. Es ist deshalb indiziert, bei solchen Untersuchungen genügend häufige Plasmaentnahmen vorzunehmen, um den Zeitpunkt der effektiven Durchmischung festhalten zu können.

\section{Die Körperflüssigkeitsräume bei Herzpatienten vor und nach Therapie}

Úber die Körperflüssigkeitsraumbestimmung beim $\mathrm{Ge}$ sunden werden wir nicht speziell berichten, dies wurde

Tab. 3

Durchmischungszeit pro Liter Gesamtkörperwasser bei Herzgesunden"

TDZ Std $/ l=$ Tritium-Wasser-Durchmischungszeit in Stunden pro Liter Gesamtkörperwasser "PCP = Primär Chronische Polyarthritis

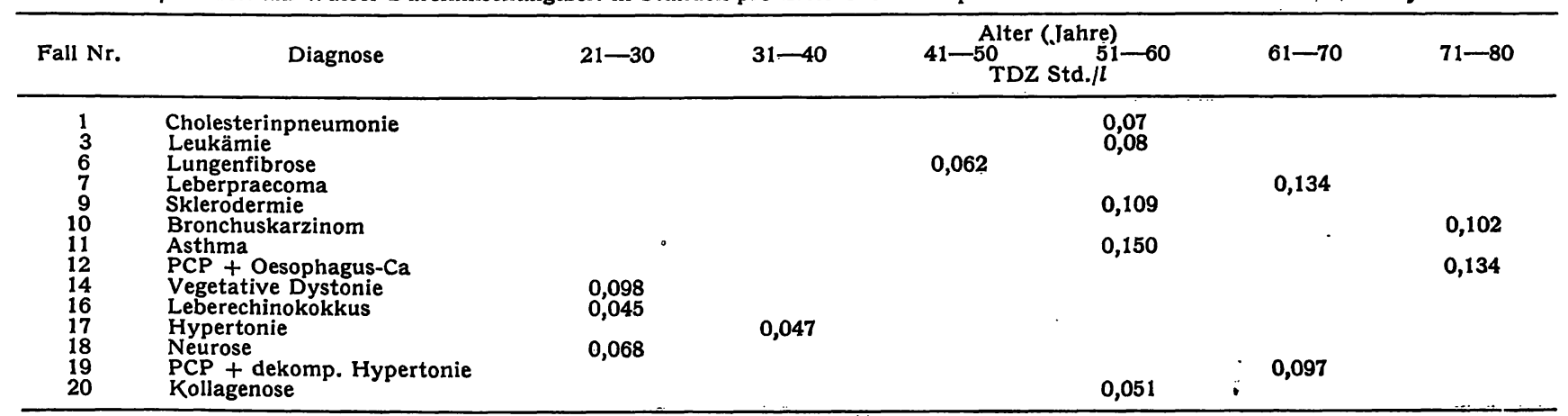




\section{Die Injektion}

\section{Technik - Praxis - Komplikationen}

\section{Von Priv.-Doz. Dr. med. Dr. med. dent. Joachim Gabka}

Groß-Oktav. VII, 205 Seiten. Mit 73, z. T. mehrfarbigen Abbildungen. 1968. Plastik flexibel DM 20,-

Es ist Gabka glänzend gelungen, die Injektionstechnik darzustellen, wobei er jeweils die topographisch-anatomische Situation schildert. Er erläutert auch die pharmaco-dynamischen Wirkungen der verschiedenen Therapeutika. Wer das Schrifttum der letzten Zeit einigermaßen kennt, weiß, daß jede Injektion mit einem gewissen Wagnis verbunden ist. Gabka, Oberarzt am RudolfVirchow-Krankenhaus in Berlin, schreibt in diesem
Buch über die Geschichte der Injektionstechnik, über die intravenöse, die intramuskuläre, die subkutane und intrakutane sowie intraarterielle Injektion. Er behandelt auch die Sterilisation und Desinfektion und äußert sich zur heutigen Rechtsprechung.

Ein gut geschriebenes, ausreichend bebildertes Buch für Studenten, Medizinalassistenten und Ärzte.

Der Medizinalbeamte

\section{Walter de Gruyter \& Co - Berlin 30}

\section{Atlas der Szintigraphie}

\section{Einführung, Technik und Praxis}

Format: $19,5 \times 27,5 \mathrm{~cm}$.

Mit 358, z. Tl. mehrfarbigen Abbildungen.

X, 294 Seiten. 1969. In Ringordner DM 190,- von

\section{Heinz Oeser \\ Werner Schumacher \\ Helmut Ernst \\ Dietrich Frost}

Es ist ein besonderes Verdienst der Verfasser, die heutige Kenntnis der szintigraphischen Untersuchungsverfahren zusammenzufassen und zu ordnen. Im vorliegenden Werk wird gleichzeitig eine Standortortung durchgeführt und der Wert der Isotopendiagnostik der verschiedenen Organsysteme abgesteckt und kritisch beurteilt.

Eine Fülle von Abbildungen - meist schwarz-weiß, nicht selten aber auch farbig - veranschaulichen die Aussage der Verfasser. Jedes Organsystem findet in der Zusammenstellung besondere Berücksichtigung.

Zentralblatt für die gesamte Neurologie

Der Atlas soll durch Ergänqungslieferungen stets auf dem neuesten Stand von Klinik und Tecbnik gebalten iverden.

Walter de Gruyter \& Co - Berlin 30 


\title{
G. Eßer
}

\section{Pfortaderhochdruck und Eiweißstoffwechsel}

\author{
Indikation und metabolische Konsequenzen porto-kavaler Anàstomosen \\ bei Leberzirrhosekranken \\ Von Priv.-Doz. Dr. med. Gregor Eßer \\ Chirurgische Universitäts-Klinik und Poliklinik Bonn (Direktor: o. Prof. Dr. A. Gütgemannn) \\ Mit 49 Abbildungen. Oktav. XII, 190 Seiten. 1969. Plastik flexibel DM 36,-
}

In der ganzen Welt steigen die Krankheiten der Leber an. Die Hepatitis steht mit an der Spitze der Infektionskrankheiten. In der Bundesrepublik übertreffen Leberschäden die Frequenz der Tuberkulosefälle. Die Anzahl der Leberkranken entspricht annähernd der der Zuckerkranken.

Die vorliegende Arbeit wurde in der Absicht geschrieben, anhand der Erfahrungen an einem einheitlichen großen Krankengut sowie aufgrund tierexperimenteller Studien eine allgemeine sowie spezielle Orientierung über Indikạtionsgirenzen und Behạndlungsprobleme der porto-kavalen Anastomose bei Leberzirrhosekranken mit Pfortaderhochdruck zu geben. Es war Bestreben des Autors, sowohl dem am Problemkreis der portalen Hypertension besonders interessierten Kliniker und Wissenschaftler als auch dem Allgemeinpraktiker einen Einblick in die Pathophysiologie des Pfortaderhochdrucks und der Pfortaderchirurgie zu vermitteln. Gleichzeitig aber sollte diese Arbeit den in der Praxis tätigen Hausärzten und Fachärzten eine Hilfe für die Auslese operativ zu behandelnder blutungsgefährdeter Leberzirrhosekranker und in der postoperativen Weiterbehandlung dieser Patienten sein.

\section{WALTER DE GRUYTER \& CO - BERLIN 30}

\section{Nierentransplantation}

Von

Prof. Dr. W. Brosig

und

Dr. R. NAGEL

Oktav. VIII, 216 Seiten.

Mit 39 Abbildungen.

1965.

Plastikeinband DM 65,-

\begin{abstract}
Die Verfasser haben unter Zugrundelegung des Weltschrifttums das gesamte Problem von frühesten Versuchen bis zu den modernsten Verfahren der Organkonșervierung und Heterotransplantation von Affennieren in einer eingehenden sachlich-abiägenden Darstellung abgehandelt. Die bisher in den Transplantationszentren der Welt durchgeführten humanen Nierentransplantationen sowie Heterotransplantationen stellen den erfolgreichen Beginn einer verantwortungsvollen, weiterzuentwickelnden Therapie für schwer nierengeschädigte Menschen mit eindeutig lebensverlängernder Wirkung dar. Von der aktuellen Thematik werden nicht nur Urologen, Nephrologen sowie Immunologen, sondern auch Internisten, Päediater und Praktiker neben dem großen Kreis der Experimentalmediziner angesprochen.
\end{abstract}

Medizinische Monatsschrift

\section{Walter de Gruyter \& Co · Berlin 30}


schon von vielen Autoren getan. Wir möchten jedoch anhand der von uns gemachten Erfahrungen, die sehr zur vorsichtigen Beurteilung mahnen, über 3 Fälle von Herzpatienten berichten, die wir vor und nach Therapie untersuchen konnten (Tab. 4).

\section{Resultate}

Fall I ( $043 \mathrm{~J}$.). Es handelt sich um einen Patienten mit rheumatischer Mitralinsuffizienz und relativer Tricuspidalinsuffizienz, der vor und nach einer Klappenoperation untersucht wurde. Die Evans-blue Durchmischungszeit hat sich postoperativ deutlich verkürzt. Das Plasmavolumen hat sich weder absolut noch auf das Körpergewicht bezogen signifikant verändert. Die Tritiumwasserdurchmischungszeit ist auf weniger als die Hälfte gesunken. Das Gesamtkörperwasser bewegt sich mit $63 \%$ bzw. $67 \%$ des Körpergewichtes prä- und postoperativ an der oberen Grenze der Norm (1-3). Die Durchmischungszeit pro Liter Körperwasser ist dagegen auf die Hälfte gesunken. Die Durchmischungszeit für das Sulfat ist ebenfalls auf die Hälfte gesunken.

Bei den Kreislaufzeiten zeigten sich der Quotient (Aufsättigungszeit/Lungen-Ohr-Zeit) AZ/LOZ praktisch unverändert (2,7 vor und 2,6 nach Operation), die AOZ (Arm-Ohr-Zeit) jedoch sank von 25 auf 12 und die LOZ fiel von 13 auf 6 Sekunden, was einer Normalisierung dieser Zeiten entspricht. Die AZ sank von 36 auf 16 Sekunden. Die Relation (Verdünnungszeit/ Konzentrationszeit) $\mathrm{VZ} / \mathrm{KZ}$ ist von 2,9 auf 1,9 gesunken, also normal geworden (Tab. 4).

Fall II (o 46 J.). Dieser Patient mit schwerer Mitralinsuffizienz mit relativer Tricuspidalinsuffizienz und geringer Aorteninsuffizienz rheumatischer Genese wurde medikamentös rekompensiert. Die Evans-blue Durchmischungszeit sank von stark erhöhten auf normale Werte. Das Plas mavolu men absolut und pro kg Körpergewicht änderte sich nicht signifikant. Die Tritiumwasserdurchmischungszeit sank absolut sowie auf das Gesamtkörperwasser bezogen auf fast die Hälfte. Das Gesamtkörperwasser ist leicht angestiegen (nicht signifikant). Die Sulfat durchmischungszeit ist um rund 30\% gesunken (Tab. 4).

Fall III (ㅇ 64 J.). Die medikamentöse Rekompensierung eines Patienten mit Aortenvitium mit vorwiegender Stenose bei stummer Anamnese in bezug auf Rheumatismus ergab folgende Änderungen der Meßwerte.
Die Evans-blue Durchmischungszeit ist von hochnormalen auf sicher pathologische Werte angestiegen. Das Plasmavolumen ist um etwa $40 \%$ gesunken, der Plasmaanteil pro kg Körpergewicht von 57 auf $45 \mathrm{ml}$. Dieser Unterschied ist nicht sicher signifikant (als sicher signifikant wurden Werte in Betracht gezogen, die bei einer Streubreite von $\pm 8 \mathrm{~m} / \mathrm{kg}$ kein Uberschneiden mehr zeigten). Die Tritiumwasserdurchmischungszeit blieb gleich, das Gesamtkörperwasser in \% des Körpergewichtes sank um 18,5\%. Die Tritiumwasserdurchmischungszeit pro Liter Körperwasser stieg von 0,135 auf 0,234 an. Die Sulfatdurchmischungszeit stieg um knapp $1 / 3$ an (Tab. 4).

\section{Diskussion}

Während sich deutlich pathologische Kreislaufzeiten normalisierten (Fall I), traten im Plasmavolumen absolut und pro $\mathrm{kg}$ Körpergewicht keine wesentlichen Änderungen auf. Gleichzeitig wurde eine Verkürzung aller Durchmischungszeiten festgestellt. Ähnliche Beobachtungen finden wir in Fall II. Bei beiden Fällen ist festzustellen, daß die Verkürzung der Durchmischungszeit von Evans blue deutlicher ist als die der anderen und zwar im Größenverhältnis 3:2. Es' besteht also keine Proportionalität $z u$ den entsprechenden Verteilungsvolumina. Im Fall III wurde eine deutliche Verlängerung der Durchmischungszeit von Evans blue bei unwesentlicher Beeinflussung der anderen festgestellt. Auf das Gesamtkörperwasser bezogen ist die Tritiumdurchmischungszeit jedoch fast auf das Doppelte gestiegen. Diese Verschlechterung der Dynamik müssen wir mit der deutlichen Verminderung des Plasmavolumens in Zusammenhang bringen (von 2,8 auf 1,7l). Bei genau gleichbleibendem Hämatokrit kann diese Änderung der Dynamik nicht mit einer Viskositätszunahme, sondern mit einer Engerstellung des gesamten Gefäßapparates erklärt werden. Die Verlängerung der Evans blue Durchmischungszeit entspricht also bei kleinerem Volumen einer noch ausgesprocheneren Verschlechterung der Hämodynamik als sie in der gemessenen Zeit zum Ausdruck kommt. Bei der Analyse und der Verlaufskontrolle einzelner Fälle kann eine gewisse Übereinstimmung zwischen Kreislauffunktion und den Durchmischungszeiten der verschiedenen Substanzen festgestellt werden, in dem Sinn, $\mathrm{da} B$ eine raschere von Evans blue mit einer entsprechenden Verkürzung der beiden anderen einhergeht.

Tab. 4

Körperflüssigkeitsräume bei Herzpatienten vor und nach Therapie $\begin{array}{lll}\text { EBDZ }=\text { Evans blue Durchmischungszeit } & \text { TDZ }=\text { Tritiumwasserdurchmischungszeit } & \text { SDZ } \\ \text { PV } & \text { GKW }=\text { Glasmavolumen } & \text { Gesamtkörperwasser }\end{array}$

\begin{tabular}{|c|c|c|c|c|c|c|c|c|c|c|c|}
\hline \multicolumn{2}{|r|}{ Fall Nr. } & Zustand & \multirow{2}{*}{$\begin{array}{l}\begin{array}{c}\text { Ge- } \\
\text { wicht } \\
\text { kg }\end{array} \\
66 \\
57,7\end{array}$} & \multirow{2}{*}{$\begin{array}{c}\begin{array}{c}\text { EBDZ } \\
\text { Min. }\end{array} \\
\begin{array}{r}30-60 \\
6-20\end{array}\end{array}$} & total & $\mathrm{m} / / \mathbf{k g}$ & \multicolumn{2}{|c|}{$\begin{array}{c}\text { TDZ } \\
\text { absolut } \\
\text { Stdn. }\end{array}$} & \multicolumn{2}{|c|}{$\underset{l}{\text { absolut }} \underset{\substack{\text { GKW } \\
\text { Kör- } \\
\text { pergew. }}}{\substack{\%\\
}}$} & $\begin{array}{l}\text { SDZ } \\
\text { absolut } \\
\text { Stdn. }\end{array}$ \\
\hline I & komb. rheum. Vitium & $\begin{array}{l}\text { vor Op. } \\
\text { nach Op. }\end{array}$ & & & $\begin{array}{l}3,5 \\
3,2\end{array}$ & $\begin{array}{l}52,2 \\
55,7\end{array}$ & $21 / 2$ & $\begin{array}{l}0,057^{*} \\
0,028\end{array}$ & $\begin{array}{l}44^{*} \\
36,4\end{array}$ & $\begin{array}{l}67 * \\
63\end{array}$ & $\begin{array}{l}1 \frac{1}{2} \\
3 / 4\end{array}$ \\
\hline II & komb. rheum. Vitium & $\begin{array}{l}\text { vor medik. Therapie } \\
\text { nach medik. Therapie }\end{array}$ & $\begin{array}{l}57,1 \\
56\end{array}$ & $\begin{array}{c}30-60 \\
<10\end{array}$ & $\begin{array}{l}3,6 \\
3,2\end{array}$ & $\begin{array}{l}62,5 \\
56,8\end{array}$ & $\begin{array}{l}4 \\
21 / 2\end{array}$ & $\begin{array}{l}0,144 \\
0,081\end{array}$ & $\begin{array}{l}27,8 \\
30,9\end{array}$ & $\begin{array}{l}49 \\
55\end{array}$ & $\begin{array}{l}3 \\
2\end{array}$ \\
\hline III & komb. Vitium & $\begin{array}{l}\text { vor medik. Therapie } \\
\text { nach medik. Therapie }\end{array}$ & $\begin{array}{l}48,6 \\
38,5\end{array}$ & $\begin{array}{l}10-20 \\
20-30\end{array}$ & $\begin{array}{l}2,8 \\
1,7\end{array}$ & $\begin{array}{l}57 \\
45\end{array}$ & $\begin{array}{l}41 / 2 \\
41 / 2\end{array}$ & $\begin{array}{l}0,135 \\
0,234\end{array}$ & $\begin{array}{l}33,4 \\
19,4\end{array}$ & $\begin{array}{l}69 \\
50,5\end{array}$ & $\begin{array}{l}3 \\
4\end{array}$ \\
\hline
\end{tabular}

* Werte ungenau 
Beim Fall IlI mit Verschlechterung der Evans blue Durchmischungszeit trat keine Verlängerung der Tritiumwasserdurchmischungszeit, die mit $4 \frac{1}{2} \mathrm{Stdn}$. schon hoch war, ein. Es ist anzunehmen, daß dies bei exsudatfreien Patienten schon einen Grenzwert darstellt. Innerhalb dieser Zeit stellt sich im vorhandenen System dank der Diffusibilität des Tritiumwassers auch bei mangelhafter Zirkulation ein Gleichgewicht her. Die Sulfatdurchmischungszeit hingegen ist trotz des kleineren Verteilungsvolumens, das durch diese Substanz erfaßt wird, unter der V'erlängerung der Evans blue Durchmischungszeit um etwa $25 \%$ angestiegen. Dies läßt auf eine geringere Diffusion dieser Substanz schließen, was angesichts der Größe des Moleküls, resp. Ions, verglichen mit Wasser, nicht erstaunlich ist. Dies würde bedeuten, daß hier der Einfluß der Zirkulationsverhältnisse auf die Sulfatdurchmischungszeit verglichen mit dem der Diffusion eine relativ größere Rolle spielt.

\section{Schlußfolgerungen}

Uber den Wert der Verdünnungsmethode zur Ermittlung der verschiedenen Körperflüssigkeitsräume haben wir uns schon am Ende des Kapitals „Nethodik" geäußert. Es sei hier nur nochmals kurz erwähnt, daß die Plasmavolumenbestimmung mit Evans-blue befriedigende Resultate ergab, während die Bestimmung des Gesamtkörperwassers und des Extrazellulärraumes mit Tritiunwasser, resp. Radiosulfat unbefriedigend sind und keinen Fortschritt gegenüber älteren Bestimmungsarten zeigen. Die Normwerte schwanken in gleich großem Naß. Es mụ daraus der Schluß gezogen werden, daß diese Räume individuell sehr verschieden sind. Die Erfassung pathologischer $\mathrm{Zu}-$ stände bei denen keine beträchtlichen Flüssigkeitsverschiebungen stattfinden, ist somit weitgehend verunmöglicht. Yerlaufsstudien, bei denen wir den Ausgangs- wert nicht kennen, haben den Nachteil, daß wir nicht wissen, wann der Patient in bezug auf seinen Flüssigkeitshaushalt seinen Normalzustand wieder erreicht hat. Diffusionsfaktoren, die wir nicht erfassen können, scheinen keine unwesentliche Rolle mitzuspielen. Uber eine große Fehlerquelle bei solchen Untersuchungen, der in der Literatur zu wenig Aufmerksamkeit geschenkt wird, haben wir speziell hingewiesen. Bei jeder Untersuchung, die nach dem Verdünnungsprinzip arbeitet, muß der Indikator homogen in dem zu messenden Milieu verteilt sein. Wir haben dies für das Evansblue im Plasmaraum überprüft. Bei gesundem Kreislauf wird die erwähnte Bedingung innerhalb 10 Minuten erfüllt, bei Kreislaufstörungen jedoch erst zwischen 30 und 60 Minuten. Anhand der von uns ermittelten Zahlen für die Tritiumwasserdurchmischungszeit können wir folgende Berechnung anstellen: für einen $75 \mathrm{~kg}$ schweren Mann mit einem Körperwassergehalt von $60 \%$ des Körpergewichtes erhalten wir bei einer Durchmischungszeit von $0,150 \mathrm{Std} . / / 63 / 4 \mathrm{Stdn}$., bei einer von 0,047 eine solche von $21 / 4$ Std. Es geht daraus eindeutig hervor, $\mathrm{da} ß$ die im allgemeinen angenommene $\mathrm{Zahl}$ von $3 \mathrm{Stdn}$. zu knapp gewählt ist. Wie wir feststellen konnten, läßt sich keine allgemeine Regel in bezug auf die Korrelation zwischen den Durchmischungszeiten von Evans-blue, Tritiumwasser und Sulfat aufstellen. Im Einzelfall kann eine Korrelation zwischen den genannten Werten bestehen. Es handelt sich also um individuell variierende $\mathbb{W}$ erte. Dies steht in Einklang mit den von anderen Autoren festgestellten starken individuellen Abweichungen in bezug auf das Gesamtkörperwasser (1). Der Wert der Einzelbestimmung ist somit eingeschränkt, da die Normalwerte beim Einzelnen ermittelt werden müssen. Nur Extremwerte können uns sichere Hinweise über die Art der Veränderung geben.

\section{Literatur}

1. Aeberhardt, A., P. Fallot, J. Canivet und J. Masson, Int. J. appl. Radiat. 2, 62 (1957). - 2. Edelaran, I. S., Amer. J. Physiol. 171, 279 (1952). - 3. Edelnan, 1. S., H. B. Halex, P. R. Schloerb, D. B. Sileldon, B. J. Frits-Hansen, G. Stoll und F. D. MOORE, Surg. Gyn. Obstetr. 95, 1 (1952). - 4. EdelMaN, I. S., J. M. Olnet, A. H. Janis, L. Brooks und E. D. MOORE, Science Washington 115, 447 (1952). - 5. MOENs, R. S., R. Busset, R. A. Coliet, C. Nagant de Deuxchatnes und R. S. МACH, Schireiz. med. IVschr. 93, 932, 965 (1963). - 6. MOORE, F. D., H. B. HALET, E. A. Bering jr. L. Brooks und I. S. Edelisan, Surg. Gjn. Obstetr. 95, 155 (1952). - 7. MoOre, D. F., J. D. MICMlurrey, H. V. Parker und I. C. Mlagnus, Mletabolism Baltimore 5, 447 (1956). - 8. Prtrs, R. F., Physiology of the kidney and body fluids. Ycarbook medical publishers, Chicago (1963). - 9. SChLOerb, P. E., J. B. Frits-HaNSEN, I. S. Edelaian, A. K. SOlomon und F. P. MOORE, J. clin. Invest. 29, 1296 (1950). 10. Schürre, E., Wasserstoffiwechsel, in Physiologische Chemie hgb. von B. Flaschenträger und E. Lehnartz II/1 S. 1554. Springer Berlin (1954). - 11. Schiwab, M. W., Th. DrssmanN und W. Schubert, Klin. W'schr. 4l, 1174 (1963). - 12. Sheppard, C. W.,
Basic principles of the tracer method; introduction to mathematical tracer kinetics. John Wilcy, New-York (1962). - 13. Siegenthaler, W., Klinische Physiologie und Pathologie des Wasser- und Salzhaushaltes mit besonderer Bcrücksichtigung der Beziehungen Aldostcron-Oedeme-Diureticn. Springer Varlag, Heidelberg (1961). - 14. UDEkwU, F. A. O., D. D. KozolL und K. A. Mleyer, J. nud. Med. 4, 60 (1963). - 15. Write, K. F., W. A. Brgler und G. R. Grove, Health Physics Pergamon Press 9, 911 (1963). - 16. Berson, S. A. und R. S. YAlow, Science Washington 121, 34 (1955). - 17. Corlove, E., Amer. J. Physiol. 176, 396 (1954). - 18. MERTz, D. P., Dic extracelluläre Flüssigkeit. Georg Thieme, Stuttgart (1962). - 19. MerTz, D. P. und H. SARRE, Klin. Wschr. 11, 868 (1963). - 20. Nichols G. jr., N. Nichols, W. B. WeIL und W. A. W WALACE, J. clin. Invest. 32, 1299 (1953). - 21. Hegglin, R., W. Rutishauser und G. KaufMaNN, E. LÜrHX und H. SChEu, Kreislaufdiagnostik mit der Farbstoffrerdünnungsnethode. Gcorg Thieme, Stuttgart (1962). 22. Rapkin, E., Packard technical Bulletin 6, 1916, La Grange, Illinois.

Prof. Dr. W. Siegenthaler Med. Univ. Poliklinik CH 8006 Zürich

Rämistr. 100 